\title{
Role of chemotherapy in nasopharyngeal carcinoma
}

\author{
Fabiola Paiar, ${ }^{1}$ Vanessa Di Cataldo, ${ }^{1}$ Giacomo Zei, ${ }^{1}$ Eleonora Monteleone Pasquetti, ${ }^{1}$ \\ Sara Cecchini, ${ }^{1}$ Icro Meattini, ${ }^{1}$ Monica Mangoni, ${ }^{1}$ Benedetta Agresti, ${ }^{1}$ Carmine lermano, ${ }^{1}$ \\ Pierluigi Bonomo, ${ }^{2}$ Giampaolo Biti ${ }^{1}$ \\ ${ }^{1}$ Radiotherapy Unit, University of Florence; ${ }^{2}$ Radiotherapy Unit, Casa di Cura S. Chiara, Florence, Italy
}

\begin{abstract}
Nasopharyngeal carcinoma (NPC) is a unique malignant head and neck cancer with clinical, demographic, and geographic features distinct from other head and neck epithelial malignancies. Non-keratinizing, poorly differentiated, and undifferentiated WHO types 2 and 3 is the most common subtypes of NPC. NPC is also characterized by its relatively high sensitivity to radiation, so that in the last decades radiotherapy (RT) has been the cornerstone of treatment. However, in the majority of cases NPC is discovered at locally advanced stage. The results are disappointing when RT alone is offered. The 5 -year survival rates have been reported to be about $34-52 \%$. The poor prognosis for advanced NPC led to increasing interests in exploring the use of chemotherapy (CT). NPC has been considered to be not only radiosensitive but also chemo-sensitive and has shown high response rate to various chemotherapeutic agents. Certainly, the treatment strategies for NPC will continue to change and evolve as a better understanding is gained of the molecular and immune mechanisms that drive this disease. We reviewed the current literature focusing on the role of CT and new-targeted agents.
\end{abstract}

\section{Introduction}

Nasopharyngeal carcinoma (NPC) is a unique malignant head/neck cancer with clinical, demographic, and geographic features distinct from other head and neck epithelial malignancies. First, NPC patients are usually younger than patients with squamous cell head and neck

Correspondence: Icro Meattini, Radiotherapy Unit, University of Florence, Largo G.A. Brambilla 3, 50134 Florence, Italy.

Tel. +39.055.7947719 - Fax: +39.055 .4379930$

E-mail: icro.meattini@unifi.it

Key words: nasopharyngeal carcinoma, chemotherapy, induction chemotherapy, targeted agents.

Received for publication: 16 June 2011.

Revision received: 9 October 2011.

Accepted for publication: 27 October 2011.

This work is licensed under a Creative Commons Attribution NonCommercial 3.0 License (CC BY-NC 3.0).

CC Copyright F. Paiar et al., 2012

Licensee PAGEPress, Italy

Oncology Reviews 2012; 6:e1

doi:10.4081/oncol.2012.e1 cancers. Secondarily, there are geographic regions in the world where NPC is endemic, such as in Southeast Asia, or Northern Africa, and parts of the Mediterranean basin. ${ }^{1}$

According to the global registry of cancer incidence, NPC ranked $11^{\text {th }}$ among all malignancies in China in 2008 , with an incidence rate of 2.8/100,000 person years in men and 1.9/100,000 person years in women. ${ }^{2}$ Non-keratinizing, poorly differentiated, and undifferentiated [World Health Organization (WHO) types 2 and 3] is the most common subtypes of NPC. ${ }^{3}$ Outside of these specific areas, the incidence of NPC remains very low, especially in Western Europe or in the United States; in these countries, the main histological version is the differentiated type that is related to tobacco use (incidence $0.5-2 / 100,000$ people per year). On the whole, keratinizing differentiated carcinoma has a reputation for less consistent primary tumor response to therapies compared with the undifferentiated subtypes, which on the other hand carry a higher risk of distant metastases and tend to have a more aggressive biological behavior.

NPC WHO type 2-3 differ from WHO type 1 with regard to their heightened sensitivity to chemotherapy (CT) and to the association with the Epstein-Barr virus (EBV) in more than $90 \%$ of cases. ${ }^{2}$ The relationship with $\mathrm{EBV}$ is of interest not only for epidemiological reasons or diagnosis, but also for the monitoring of patients, prognosis and therapeutic strategies. ${ }^{4-6}$

NPC is also characterized by its relatively high sensitivity to radiation, so that in the last decades radiotherapy (RT) has been the cornerstone of treatment. Patients presenting with early-stage disease (T1$2 \mathrm{a} ; \mathrm{N} 0$; M0) are a minority and may be effectively treated with exclusive RT. However, in the majority of cases NPC is discovered at locally advanced stage. The results are disappointing when RT alone is offered. The 5-year survival rates have been reported to be about 34 $52 \%{ }^{7-14}$ The poor prognosis for advanced NPC led to increasing interests in exploring the use of CT. NPC has been considered to be not only radiosensitive but also chemo-sensitive and has shown high response rate to various chemotherapeutic agents. ${ }^{15-18}$

The integration of CT with standard RT in the treatment of patients with non-metastatic Stage III/IV NPC is based on the following rationale/hypotheses: i) to minimize the risk of distant recurrence through eradication of micro-metastases; ii) to enhance the effects of radiation through synergistic agents (radio-sensitizers); iii) to facilitate planning of RT and to improve local disease control by reducing the tumor volume prior to irradiation.

\section{Concurrent-adjuvant chemo-therapy for locally advanced nasopharyngeal carcinoma}

Today CT is usually integrated into the RT approach for most patients with non-metastatic Stage III/IV NPC. In particular, since the publication of the results of the Intergroup Study $0099,{ }^{19}$ concomitant chemo-radiotherapy (CRT) and adjuvant CT has been widely accepted as standard in the treatment of patients with stage III and IV NPC. The 
Intergroup-0099 trial (INT-0099) is the first phase III randomized trial comparing CRT versus exclusive RT in patients with locally advanced NPC. It was coordinated by the Southwest Oncology Group (SWOG), with the participation of Radiation Therapy Oncology Group (RTOG) and Eastern Cooperative Oncology Group (ECOG). The patients were stratified by tumor stage, nodal stage, performance status, and histology into CRT arm or RT arm.

RT was administered in both arms: 1.8-2.0 Gy per fraction was delivered for 35-39 fractions to a total dose of 70 Gy. Patients in the CRT arm received cisplatin $100 \mathrm{mg} / \mathrm{m}^{2}$ on days 1,22 , and 43 during $\mathrm{RT}$, followed by adjuvant cisplatin $80 \mathrm{mg} / \mathrm{m}^{2}$ on day 1 and 5 -fluorouracil $1000 \mathrm{mg} / \mathrm{m}^{2}$ on days 1-4, every 4 weeks, for three cycles. The study found a highly significant 3-year overall survival (OS) advantage for the CRT arm ( $76 \%$ vs $46 \%, \mathrm{P}<0.001$ ). In the updated report, ${ }^{20}$ the 5 -year results continued to show a statistically significant difference in OS: $67 \%$ in the CRT arm, compared with $37 \%$ in the RT arm $(\mathrm{P}=0.001)$. In addition, improved disease-free survival (DFS) and decreased both loco-regional failure and distant metastasis occurrence were also observed in the CRT arm. However, the reproducibility of the Intergroup findings and, more specifically, the benefit of its experimental regimen in regions of endemic NPC were questioned.,21 Many Asian institutions initiated similar phase III studies to evaluate the role of concurrent CRT in NPC in their patient populations. ${ }^{22-26}$ In 2002, Chan et al..$^{22}$ from Hong Kong were the first Asian group to publish the results of their concurrent CRT study, and, in their initial publication, no improvement was found in 2-year progression-free survival (PFS) with the addition of weekly cisplatin $\left(40 \mathrm{mg} / \mathrm{m}^{2}\right)$ administered concurrently with conventional RT. In a recent update, ${ }^{27}$ however, the Authors reported the concurrent CRT arm to be associated with improvements in both 5-year PFS and OS. In addition, Lin et al. ${ }^{23}$ from Taiwan, Kwong et al. ${ }^{24}$ from Hong Kong, Wee et al. ${ }^{25}$ from Singapore, Lee et al. ${ }^{28,29}$ from Hong Kong and Chen et al. from Guangzhou, ${ }^{30}$ have recently published or reported in abstract form the results of their trials. In particular the results of the latter three trials not only confirmed the findings of the 00-99 study, but also showed a different gain in survival. The absolute increase in 5-year OS ranged from $40 \%$ in the Intergroup- 0099 Study $^{19}$ to $18 \%$ by Wee, ${ }^{25} 10 \%$ by Chen ${ }^{30}$ and $4 \%$ in Lee. ${ }^{29}$ One major question regarding the design of the Intergroup-0099 regimen ${ }^{19}$ is the contribution of the adjuvant phase, because the available randomized trials ${ }^{24,31,32}$ and two recent meta-analysis ${ }^{33,34}$ showed that adjuvant CT per se had no statistically significant impact for all endpoints.

To our knowledge, no randomized trial to date has compared the efficacy of CRT versus CT alone. The only available data are from a retrospective comparison by Cheng et al. ${ }^{35}$ who showed that inclusion of the adjuvant phase was beneficial for patients with intermediate risk (T2b3N0-2M0); in this study, the 5-year OS by concurrent-adjuvant CT was $84 \%$, compared with $63 \%$ for RT or concurrent CRT alone ( $\mathrm{P}=0.005)$. Controversy continues about the optimal schedule and the duration of CT, including the types of drugs and their doses.

\section{Meta-analysis}

In an attempt to assess the potential benefit of adding CT to standard RT in locally advanced NPC, some authors conducted meta-analysis.

The meta-analysis by Huncharak et al. ${ }^{36}$ reported pooled results of six randomized trials..$^{27,37-41}$ All trials compared standard radical external beam RT (control arm) plus CT delivered either adjuvantly, neoadjuvantly, or concurrently with RT. Pooling all six studies using DFS/PFS as endpoints, data showed that the addition of CT to RT increased DFS/PFS by $37 \%$ at 2 years, $40 \%$ at 3 years, and $34 \%$ at 4 years after treatment. Three- and 4-year OS was increased by $19 \%$ and $21 \%$, respectively, with the data for 4-year survival being statistically significant. The addition of CT to standard radical RT for loco-regionally advanced NPC increases both DFS/PFS and OS by $19-40 \%$ at 2 to 4 years after treatment, depending on the endpoint of interest. Likewise, the summary relative risk for $\mathrm{OS}$ at 2 years after treatment with the addition of CT to the treatment regimen was 0.80 (0.63-1.02), reflecting a $20 \%$ increase in 2 -year survival.

In an update of the analysis significant heterogeneity was identified across trials, and the authors concluded that the trial by Al-Sarraf $e t$ al. was the source of the heterogeneity. The authors reported that CRT significantly improved 2-4 year DFS by approximately $35-40 \%$ over RT alone. ${ }^{19,39} \mathrm{CRT}$ did not improve two- and three-year OS in patients over RT alone; however, four-year results achieved statistical significance. We have to be aware of the distribution of WHO histologic type in the six trials. The majority of WHO histology was type II and III in all trials except the INT-0099 in which type I accounted for 20-30\%. Epidemiologic study have identified WHO histologic type as an independent prognostic factor for survival in NPC. ${ }^{42,43}$

Type II and III tumors are more often controlled by RT than type I tumor, and the 5 -year OS rate is significantly better for type II and III than type I (51\% vs 6\%). ${ }^{44}$ To avoid biased results potentially caused by tumor histology, a sensitivity study performed by excluding the INT-099 from the meta-analysis and summary odds ratio (OR p) was recalculated. However, a statistically significant result with an OR p of $0.73(95 \%$ CI, 0.59-0.91) was found despite the omission of the INT-0099 from the analysis, suggesting WHO type I tumors did not result in a strong impact on outcome. ${ }^{36}$

The meta-analysis reported by Langendijk et al. ${ }^{33}$ employed the hazard ratio (HR) to assess OS across 10 trials with a total of 2450 patients. Results from this analysis indicate a small but significant OS benefit in favor of CRT when compared with RT alone. The greatest magnitude of effect was detected for concurrent CT regimens (HR=0.48; 95\% CI, 0.32-0.72).

The third meta-analysis, ${ }^{45}$ published as an abstract, pooled results from 78 randomized trials (9279 patients) that were identified in English and non-English-based databases. Results were expressed using the odds ratio and the random effects model. Significant differences in OS and DFS were detected in favor of CRT when compared with RT alone. When sensitivity analyses were conducted to remove studies rated as low quality, the results remained unchanged. The greatest magnitude of effect in OS was detected with concurrent plus adjuvant $\mathrm{CT}(\mathrm{OR}=0.33 ; 95 \% \mathrm{CI}, 0.20$ to 0.56 ), however neoadjuvant and concurrent CT also produced significant improvements over RT alone. When Chinese language trials were excluded in a sensitivity analysis, the magnitude of effect associated with neoadjuvant CT became of borderline significance.

The meta-analysis of CT in nasopharyngeal carcinoma study of the MAC-NPC Collaborative Group is the only meta-analysis that used an individual patient data design. ${ }^{34}$ As reported in 2006, the MAC-NPC study included 8 randomized trials, which had completed accrual before end of 2001 and thus excluded the more recent trials from Asia. In the meta-analysis, there were 4 trials that investigate induction CT (plus adjuvant CT in 1 trial), 3 trials that investigate concurrent CRT (plus adjuvant CT in 2 trials) and 1 trial that investigate adjuvant CT alone. Overall, an absolute survival benefit of $6 \%$ at 5 year from addition of CT was observed (from 56-62\%).

A significant interaction was observed between the timing of CT and OS, with the highest benefit resulting from concurrent CRT.

More recently, Zhang et al. ${ }^{46}$ performed a first meta-analysis of concurrent CT versus RT alone in NPC treatment, which included six, randomized controlled trials with 1483 patients done only in endemic areas. Published in an abstract form, the analysis found an HR of 0.65 (95\% CI, 0.51-0.83), 0.72 (95\% CI, 0.59-0.87), and 0.72 (95\% CI, 0.540.95 ) in favor of CRT for 2-, 3-, and 5-year OS, respectively. In addition, CRT was also associated with both improvement of loco-regional recur- 
rence rate and distant metastasis rate. Apparently, the study may allow establishing concurrent CRT as standard practice for NPC patients with locally advanced disease in endemic regions.

Concluding, the results of meta-analyses showed that concomitant CT in addition to RT is probably the most effective way to improve OS in NPC. Neoadjuvant CT significantly reduced the risk of loco-regional recurrence and distant metastases; therefore, the addition of induction CT to concomitant CRT warrants additional investigation. The exact role of adjuvant CT remains unclear because it has not been adequately tested and the compliance is difficult.

\section{Induction chemotherapy}

In the early-randomized trials, induction CT is the most often studied combination. ${ }^{47-49}$ The rationale for induction CT is to reduce tumor load of loco-regional disease before start of RT and also early use of systemic treatment for eradication of micro-metastases. The role of neoadjuvant CT before RT or CRT is a matter of outstanding interest.

Initially, RT alone was considered as the control arm in randomized trials. Ma et al. ${ }^{39}$ showed the lack of significant survival benefit with the addition of neoadjuvant CT (cisplatin, bleomycin, and 5-FU) to standard radiation therapy in patients with loco-regionally advanced NPC. Al-Amro et al., ${ }^{50}$ tested induction CT (cisplatin and epirubicin) followed by a radical course of RT with three cycles of concurrent cisplatin in the same patient population. This study, carried out in 110 patients, showed encouraging results in terms of safety and effectiveness. The only induction trial that showed a significant improvement of DFS was the International Nasopharyngeal Carcinoma Study Group, ${ }^{40}$ using combination of bleomycin, epirubicin and cisplatin. However, there was no improvement in OS. This discrepancy in findings for disease-free and OS may be due to the increased treatment-related death among patients on induction CT which would offset the benefit of CT in reducing disease-related death. The impressive activity of the taxanes, namely docetaxel and paclitaxel, against HNSCC ${ }^{48,49,51}$ sparked new interest in induction CT also in nasopharyngeal cancer. Paclitaxel is a compound derived from the bark of the Pacific yew, and causes cell death by binding to beta-tubulin resulting in cell cycle arrest at G2-M phase. Docetaxel, a semi-synthetic taxoid, has a similar mechanism of action. The combination of paclitaxel and carboplatin was shown to have a high response rate (75\%) in patients with metastatic NPC, 52 and has demonstrated encouraging activity and safety profiles as a neoadjuvant treatment of NPC. ${ }^{53}$ A number of uncontrolled phase II studies, have explored neoadjuvant taxanes-based CT followed by CRT in NPC. The results have been encouraging, and toxicity has been acceptable. Bossi et al..$^{53}$ presented the data of a study of docetaxel, cisplatin, 5-FU as induction CT followed by concomitant cisplatin/RT. After completion of treatment, complete response (CR) and partial response (PR) were observed in 78 and $20 \%$ of the patients, respectively. Recently they published the first study to evaluate TPF induction CT as a part of a sequential regimen including IMRT concurrent with high-dose cisplatin CT in 30 patients with locally advanced NPC. ${ }^{54}$ All patients showed a PR after induction CT and a high rate of CR after completing treatment (28/30; $93 \%$ of patients). At a median follow-up of 35 months a CR was still evident in 24 (80\%) patients, while 6 (20\%) experienced recurrence of disease. Three-year PFS and OS were $79 \%$ and $87 \%$, respectively. During CRT, neutropenia (40\%) and mucositis (43\%) were the most frequent grade 3-4 adverse events. Mean dose of IMRT was $68.8 \mathrm{~Gy}$. Their results suggest that this treatment schedule has an acceptable toxicity profile, allows for full RT delivery and has a promising efficacy. Noteworthy, the induction TPF used in this study allowed a reduction in the doses of 5-FU when compared with the TPF developed in Europe.

Bae et al. ${ }^{55}$ published the results of a phase II trial on 33 patients treated with three cycles of induction CT (TPF Docetaxel $70 \mathrm{mg} / \mathrm{m}^{2}$, cis- platin $75 \mathrm{mg} / \mathrm{m}^{2} 5$-FU $1000 \mathrm{mg} / \mathrm{m}^{2}$ ). After induction CT, cisplatin was given at a dose of $100 \mathrm{mg} / \mathrm{m}^{2}$, every 3 weeks with RT. They obtained $15.2 \%$ of CR, and $81.8 \%$ of PR. The 3 -year PFS was $75.6 \%$ and the 3 -year OS was $86.1 \%$. Neutropenia (72.7\%), febrile neutropenia (9.1\%), and nausea (9.1\%) were the most severe toxicities (grade 3-4) during induction CT, and mucositis (39.4\%), fatigue (15.2\%), and nausea (9.1\%) were the most common toxicities (grade 3-4) during CRT.

Hui et al..$^{56}$ have recently published the results of a randomized phase II trial in which stage III to IVB NPC untreated earlier were randomly assigned to receive either neoadjuvant docetaxel and cisplatin for two cycles followed by CRT, or CRT alone. The neoadjuvant regimen turned out to be active and well tolerated with a manageable toxicity profile that allowed subsequent delivery of full dose CRT.

The multicentre randomized trial by the Asian-0ceanian Clinical Oncology Association Nasopharynx Cancer Study Group, ${ }^{37}$ showed no significant difference in DFS or OS between the two treatment arms (3-year RFS rate: $48 \%$ in the neoadjuvant CT arm vs. $42 \%$ in the RT arm, $\mathrm{P}=0.45$; 3 -year $0 \mathrm{~S}$ rate: $78 \%$ vs $71 \%, \mathrm{P}=0.57$ ). A trend of improved relapse free survival favoring the CT arm was observed (3-year RFS rate: $58 \%$ vs $46 \%, \mathrm{P}=0.053$ ). In the subgroup of 49 patients with bulky neck lymph nodes, improved RFS (3-year RFS rate: $63 \%$ vs $28 \%$, $\mathrm{P}=0.026$ ) and $\mathrm{OS}$ ( 3 -year $\mathrm{OS}$ rate: $73 \%$ vs $37 \%, \mathrm{P}=0.057$ ) were observed, favoring the CT arm.

Hareyama et al. ${ }^{57}$ found a trend toward improved OS or DFS favoring the CT arm (5-year OS rate, $60 \%$ vs 48\%; 5 -year DFS, $55 \%$ vs 43\%), although the difference was not significant.

\section{Molecular targeted agents}

Several molecular targets have been identified in NPC. Expression or over-expression of epidermal growth factor receptor (EGFR), vascular endothelial growth factor (VEGF), c-KIT, and c-erbB-2 (HER2) has been seen in NPC. ${ }^{58}$ Inhibition of EGFR is rational, as it is overexpressed in up to $80-90 \%$ of head and neck cancers including NPC and is associated with an adverse outcome. ${ }^{59-66}$ Examples of EGFR inhibitors include mAbs against the extracellular domain of this receptor (e.g., cetuximab and panitumumab) and receptor tyrosine kinase inhibitors (TKIs) that target the intracellular domain (e.g., gefitinib and erlotinib). Given that EGFR dimerizes preferentially to the human epidermal receptor 2 (HER-2) and this heterodimer may potentiate receptor signaling and resistance to EGFR inhibitors, molecules that target both of these pathways, such as lapatinib, have also been investigated. ${ }^{67}$

In NPC, the use of anti-EGFR therapy has also been tested, though not to the same extent as head and neck squamous cell carcinoma (HNSCC). In the metastatic/recurrent (MRHNSCC) disease setting, the use of single anti-EGFR agent has been disappointing. Two-phase II trials ${ }^{68,69}$ have been reported to date, utilizing gefitinib, with no evidence of response, while stable disease ranged from 11-19\%. A phase II trial $^{69}$ in this setting consisting of combination of cetuximab and carboplatin, however, showed more promising results with an ORR of $12 \%$ and with $48 \%$ of patients having stable disease. In another trial ${ }^{70}$ the role of maintenance therapy with erlotinib after 6 cycles of platinumgemcitabine CT has been recently presented. In this phase II study, erlotinib was found not to be effective as a maintenance therapy, with the majority of patients progressed on first assessment while on erlotinib. These results, akin to the trials of targeted therapy in RMHNSCC, are very difficult to gauge in terms of efficacy, largely due to the heterogeneity of the trial population.

In the localized disease setting for NPC, there has been a phase II trial assessing platinum-based CRT and cetuximab. Preliminary efficacy data from this study, which consisted of 12 evaluable patients, were very encouraging, with $\mathrm{CR}$ seen in 10 and $\mathrm{PR}$ in the remainder of the cohort. ${ }^{71}$ However, similar to combination of targeted therapy and 
chemo-radiation in HNSCC, there was significant toxicity reported in this study, with up to $85 \%$ of patients experiencing grade 3 or 4 mucositis and leucopenia. There is also ample evidence demonstrating the central role of angiogenesis in head and neck cancers. Up to $90 \%$ of HNSCC and NPC express angiogenic factors such as VEGF. Increased vascular endothelial growth factor-A (VEGF-A) expression has been associated with poor prognosis in squamous cell carcinoma of the head and neck. ${ }^{72-81}$

VEGF has been shown to play an important role in lymph node metastasis through the induction of angiogenesis in nasopharyngeal carcinoma. ${ }^{75}$ In another study, Qian et al. ${ }^{76}$ has shown that the levels of serum VEGF were significantly elevated in 65 patients with metastatic nasopharyngeal carcinoma. A meta-analysis of twelve studies evaluating VEGF-A expression in 1002 patients with HNSCC,, 80 showed that positive VEGF staining was associated with an almost two-fold higher risk of death at 2 years. Lastly, over-expression of VEGF was seen in $67 \%$ of nasopharyngeal cases and the higher expression of VEGF in EBV positive tumors was related to higher rate of recurrence, nodal positivity, and lower survival. ${ }^{79} \mathrm{~A}$ recent pilot study by Druzgal et al., ${ }^{81}$ analyzed the pre- and post-treatment serum levels of cytokines and angiogenesis factors as markers for outcome in patients with head and neck cancer. With a median follow-up of 37 months, patients were more likely to remain disease free when the VEGF level decreased post-treatment versus those who continued to have increased VEGF levels after treatment. In this study, $7 \%$ of the patients had NPC.

Given that high VEGF expression is associated with a worse outcome, inhibition of this molecule or its receptor has served an attractive platform in the treatment of head and neck cancers.

In NPC, there are a very few of trials assessing the role of AAT, and the results are commonly disappointing. In one phase II trial ${ }^{79}$ seven patients with recurrent or metastatic NPC were treated with sorafenib; the median time to treatment progression was 3.2 months, with an associated OS of 7.7 months. Currently, the RTOG has completed accrual to a phase II trial (RTOG 0615), assessing the role of bevacizumab added to concurrent CRT and adjuvant cisplatin and 5-FU in locally advanced/advanced NPC. ${ }^{77,81}$

\section{Conclusions}

Based on large number of randomized controlled trials and metaanalyses, there are sufficient evidences to support CRT should be adopted as the standard of care for locally advanced NPC, not only in endemic areas, but also in non-endemic areas. Concomitant CRT has shown statistically significant improvement in OS and DFS for all histological types of locally advanced NPC, and achieving 5 -year OS rates of about $70 \%$ in patients with non-metastatic stage III and IV disease. Cisplatin-containing regimens were most widely studied and have been conclusively proven active in this disease. However, the CT regimen varied markedly among those trials, and the optimal regimen and scheduling remains to be determined. Incorporation of newer, less toxic and more effective anticancer agents such as taxanes, or molecular targeted agents in combined modality regimens are being assessed in locally advanced NPC to improve the outcomes and to reduce the related treatment toxicity. Certainly, the treatment strategies for NPC will continue to change and evolve as a better understanding is gained of the molecular and immune mechanisms that drive this disease.

\section{References}

1. Yu MC, Yuan JM. Epidemiology of nasopharyngeal carcinoma. Semin Cancer Biol 2002;12:421-9.

2. Cao SM, Simons MJ, Qian CN. The prevalence and prevention of nasopharyngeal carcinoma in China. Chin J Cancer 2011;30:114-9.

3. World Health Organization. International classification of tumors: histological typing of upper respiratory tract tumors. Geneva: World Health Organization; 1991.

4. Lo YMD, Chan LYS, Lo CKW, et al. Quantitative analysis of cell-free Epstein-Barr virus DNA in plasma patients with nasopharyngeal carcinoma. Cancer Res 1999;59:1188-91.

5. Lo YMD, Chan LYS, Chan ATC, et al. Quantitative and temporal circulating cell-free Epstein-Barr virus DNA and tumor recurrence in nasopharyngeal carcinoma. Cancer Res 1999;59:5452-5.

6. Lo YMD, Chan ATC, Chan LYS, et al. Molecular prognostication of nasopharyngeal carcinoma by quantitative analysis of circulating Epstein-Barr virus DNA. Cancer Res 2000;60:6878-81.

7. Marks JE, Philips JL, Menck HR. The national cancer database report on the relationship of race and national origin to the histology of nasopharyngeal carcinoma. Cancer 1998;83:582-8.

8. Jiong L, Berrino F, Coebergh JWW. Variation in survival for adults with nasopharyngeal cancer in Europe, 1978-1989. Eur J Cancer 1998;34:2162-6.

9. Hsu MM, Tu SM. Nasopharyngeal carcinoma in Taiwan: clinical manifestations and results of therapy. Cancer 1983;52:362-8.

10. Lee AWM, Poon YF, Foo W, et al. Retrospective analysis of 5037 patients with nasopharyngeal carcinoma treated during 1976-1985: overall survival and patterns of failure. Int J Radiat Oncol Biol Phys 1992;23:261-70.

11. Lu H, Yao M. The current status of intensity-modulated radiation therapy in the treatment of nasopharyngeal carcinoma. Cancer Treat Rev 2008;34:27-36.

12. Chen WZ, Zhou DL, Luo KS. Long-term observation after radiotherapy for nasopharyngeal carcinoma (NPC). Int J Radiat Oncol Biol Phys 1989;16:311-4.

13. Qin DX, Yu YH, Yan JH, et al. Analysis of 1379 patients with nasopharyngeal carcinoma treated by radiation. Cancer 1988;61:1117-24.

14. Sanguineti G, Geara FB, Garden AS, et al. Carcinoma of the nasopharynx treated by radiotherapy alone: determinants of local and regional control. Int J Radiat Oncol Biol Phys 1997;37:985-96.

15. Ngan RK, Yiu HH, Lau WH, et al. Combination gemcitabine and cisplatin chemotherapy for metastatic or recurrent nasopharyngeal carcinoma: report of a phase II study. Ann Oncol 2002;13:1252-8.

16. Taamma A, Fandi A, Azli N, et al. Phase II trial of chemotherapy with 5- fluorouracil, bleomycin, epirubicin, and cisplatin for patients with locally advanced, metastatic, or recurrent undifferentiated carcinoma of the nasopharyngeal type. Cancer 1999;86:11018.

17. Siu LL, Czaykowski PM, Tannock IF. Phase I/I study of the CAPBLE regimen for patients with poorly differentiated carcinoma of the nasopharynx. J Clin Oncol 1998;16:2514-21.

18. Fnadi A, Altun M, Azli N, et al. Nasopharyngeal cancer, epidemiology, staging and treatment. Semin Oncol 1994;21:382-97.

19. Al-Sarraf M, Leblanc M, Giri PG, et al. Chemoradiotherapy versus radiotherapy in patients with nasopharyngeal cancer: Phase III randomized Intergroup study 0099. J Clin Oncol 1998;16:1310-7.

20. Al-Sarraf M, LeBlanc M, Giri PG, et al. Superiority of 5-year survival with chemoradiotherapy vs. radiotherapy in patients with locally advanced nasopharyngeal cancer. Intergroup 0099 phase III study: final report abstract. Proc Am Soc Clin Oncol 2001;20:227a.

21. Cooper JS, Lee H, Torrey M, Hochster H. Improved outcome second- 
ary to concurrent chemoradiotherapy for advanced carcinoma of the nasopharynx: Preliminary corroboration of the intergroup experience. Int J Radiat Oncol Biol Phys 2000;47:861-6.

22. Chan AT, Teo PM, Ngan RK, et al. Concurrent chemotherapy-radiotherapy compared with radiotherapy alone in locoregionally advanced nasopharyngeal carcinoma: progression-free survival analysis of a phase III randomized trial. J Clin Oncol 2002;20:203844.

23. Lin JC, Jan JS, Hsu CY, et al. Phase III study of concurrent chemoradiotherapy versus radiotherapy alone for advanced nasopharyngeal carcinoma: positive effect on overall and progression-free survival. J Clin Oncol 2003;21:631-7.

24. Kwong DL, Sham JS, Au GK, et al. Concurrent and adjuvant chemotherapy for nasopharyngeal carcinoma: a factorial study. J Clin Oncol 2004;22:2643-53.

25. Wee J, Tan EH, Tai BC, et al. Phase III randomized trial of radiotherapy versus concurrent chemo-radiotherapy followed by adjuvant chemotherapy in patients with AJCC/UICC (1997) stage 3 and 4 nasopharyngeal cancer of the endemic variety. J Clin Oncol 1997;22(14S):5500.

26. Lee AW, Lau WH, Tung SY, et al. Prospective randomized study on therapeutic gain achieved by addition of chemotherapy for T1-4N23M0 nasopharyngealcarcinoma (NPC). J Clin Oncol 2004:22(14S):5506.

27. Chan AT, Ngan R, Teo P, et al. Final results of a phase III randomized study of concurrent weekly cisplatin-RT versus RT alone in locoregionally advanced nasopharyngeal carcinoma (NPC). J Clin Oncol 2004;22(14S):5523.

28. Lee AW, Lau WH, Tung SY, et al. Preliminary results of a randomized study on therapeutic gain by concurrent chemotherapy for regionally advanced nasopharyngeal carcinoma: NPC-9901 trial by the Hong Kong Nasopharyngeal Cancer Study Group. J Clin Oncol 2005;23:6966-75.

29. Lee AW, Tung SY, Chua DT, et al. Randomized trial of radiotherapy plus concurrent-adjuvant chemotherapy vs radiotherapy alone for regionally advanced nasopharyngeal carcinoma. J Natl Cancer Inst 2010;102:1188-98.

30. Chen Y, Liu MZ, Liang SB, et al. Preliminary results of a prospective randomized trial comparing concurrent chemoradiotherapy plus adjuvant chemotherapy with radiotherapy alone in patients with locoregionally advanced nasopharyngeal carcinoma in endemic regions of china. Int J Radiat Oncol Biol Phys 2008;71:1356-64.

31. Rossi A, Molinari R, Boracchi P, et al. Adjuvant chemotherapy with vincristine, cyclophosphamide and doxorubicin after radiotherapy in local-regional nasopharyngeal cancer: results of a 4-year multicenter randomized study. J Clin Oncol 1988;6:1401-10.

32. Chi KH, Chang YC, Guo WY, et al. A phase III study of adjuvant chemotherapy in advanced nasopharyngeal carcinoma patients. Int J Radiat Oncol Biol Phys 2002;52:1238-44.

33. Langendijk JA, Leemans ChR, Buter J, et al. The Additional Value of Chemotherapy to Radiotherapy in Locally Advanced Nasopharyngeal Carcinoma: A Meta-Analysis of the Published Literature. J Clin Oncol 2004;22:4604-12.

34. Baujat B, Audry H, Bourhis J, et al. Chemotherapy in locally advanced nasopharyngeal carcinoma: an individual patient data meta-analysis of eight randomized trials and 1753 patients. Int $\mathrm{J}$ Radiat Oncol Biol Phys 2006;64:47-56.

35. Cheng SH, Tsai YC, Yen KL, et al. Prognostic significance of parapharyngeal space venous plexus and marrow involvement: potential landmarks of dissemination for stage I-III nasopharyngeal carcinoma. Int J Radiat Oncol Biol Phys 2005;61:456-65.

36. Huncharek M, Kupelnick B. Combined chemoradiation versus radiation therapy alone in locally advanced nasopharyngeal carcinoma: results of a meta-analysis of 1528 patients from six randomized tri- als. Am J Clin Oncol 2002;25:219-23.

37. Chua DT, Sham JS, Choy D, et al. Preliminary report of the AsianOceanian Clinical Oncology Association randomized trial comparing cisplatin and epirubicin followed by radiotherapy versus radiotherapy alone in the treatment of patients with locoregionally advanced nasopharyngeal carcinoma: Asian- Oceanian Clinical Oncology Association Nasopharynx Cancer Study Group. Cancer 1998;83:2270-83.

38. Chan AT, Teo PM, Leung TW, et al. A prospective randomized study of chemotherapy adjunctive to definitive radiotherapy in advanced nasopharyngeal carcinoma. Int J Radiat Oncol Biol Phys 1995; 33:569-577.

39. Ma J, Mai HQ, Hong MH, et al. Results of a prospective randomized trial comparing neoadjuvant chemotherapy plus radiotherapy with radiotherapy alone in patients with locoregionally advanced nasopharyngeal carcinoma. J Clin Oncol 2001;19:1350-7.

40. International Nasopharynx Cancer Study Group. Preliminary results of a randomized trial comparing neoadjuvant chemotherapy (cisplatin, epirubicin, bleomycin) plus radiotherapy vs. radiotherapy alone in stage IV undifferentiated nasopharyngeal carcinoma: a positive effect on progression free survival. Int $\mathbf{J}$ Radiat Oncol Biol Phys 1996;35:463-9.

41. Decker DA, Drelichman A, Al-Sarraf M, et al. Chemotherapy for nasopharyngeal cancer: A ten year experience. Cancer 1983;52: 602-5.

42. Levine PH, Connelly RR, Easton JM. Demographic patterns for nasopharyngeal carcinoma in the United Stated. Int J Cancer 1980; 26:741-8.

43. Burt RD, Vaughan TL, Mcknight B. Descriptive epidemiology and survival analysis of nasopharyngeal carcinoma in the United States. Int J Cancer 1992;52:549-56.

44. Reddy SP, Rasian WF, Gooneratne S, et al. Prognostic significance of keratinizing in nasopharyngeal carcinoma. Am J Otolaryngol 2005; $16: 103-8$.

45. Thephamongkhol K, Zhou J, Browman G, et al. Chemo-radiotherapy versus radiotherapy alone for nasopharyngeal carcinoma: A meta-analysis of 78 randomized controlled trials (RCTs) from English and non-English databases. J Clin Oncol 2004;22 (S14):5522.

46. Zhang L, Zhao C, Ghimire BR, et al. The role of concurrent chemoradiation in the treatment of locally advanced nasopharyngeal carcinoma among endemic area: a meta-analysis of the phase III randomized trials abstract. Proc Am Soc Clin Oncol 2008;26:6032.

47. Vermorken JB, Remenar E, Van Herpen C, et al. Cisplatin, fluorouracil, and docetaxel in unresectable head and neck cancer. $\mathrm{N}$ Engl J Med 2007;357:1695-704.

48. Posner M, Hershok DM, Blajman CR, et al. Cisplatin and fluorouracil alone or with docetaxel in head and neck cancer. $\mathrm{N}$ Engl $\mathrm{J}$ Med 2007;357:1705-15.

49. Hitt R, Lopez-Pousa A, Martinez-Trufero J, et al. Phase III study comparing cisplatin plus fluorouracil to paclitaxel, cisplatin, and fluorouracil induction chemotherapy followed by chemoradiotherapy in locally advanced head and neck cancer. J Clin Oncol 2005;23:8636-45.

50. Al-Amro A, Al-Rajhi N, Khafaga Y, et al. Neoadjuvant chemotherapy followed by concurrent chemo-radiation therapy in locally advanced nasopharyngeal carcinoma. Int J Radiat Oncol Biol Phys 2005;62: 508-13.

51. Tan EH, Khoo KS, Wee J, et al. Phase II trial of a paclitaxel and carboplatin combination in Asian patients with metastatic nasopharyngeal carcinoma. Ann Oncol 1999;10:235-7.

52. Chan AT, Ma BB, Lo YM, et al. Phase II study of neoadjuvant carboplatin and paclitaxel followed by radiotherapy and concurrent cis- 
platin in patients with locoregionally advanced nasopharyngeal carcinoma: Therapeutic monitoring with plasma Epstein-Barr virus DNA. J Clin Oncol 2004;22:3053-60.

53. Bossi P, Parolini D, Bergamini C, et al. TPF induction chemotherapy (CT) followed by concomitant cisplatin/ radiotherapy (cCTRT) in locally advanced nasopharyngeal cancer (LANPC). J Clin Oncol 2009;27(S15):6046.

54. Bossi P, Orlandi E, Bergamini C, et al. Docetaxel, cisplatin and 5fluorouracil-based induction chemotherapy followed by intensitymodulated radiotherapy concurrent with cisplatin in locally advanced EBV-related nasopharyngeal cancer. Ann Oncol 2011. [Epub ahead of print].

55. Bae WK, Hwang JE, Shim HJ, et al. Phase II study of docetaxel, cisplatin, and 5-FU induction chemotherapy followed by chemoradiotherapy in locoregionally advanced nasopharyngeal cancer. Cancer Chemother Pharmacol 2010;65:589-95.

56. Hui EP, Ma BB, Leung SF, et al. Randomized phase II trial of concurrent cisplatin-radiotherapy with or without neoadjuvant docetaxel and cisplatin in advanced nasopharyngeal carcinoma. J Clin Oncol 2009;27:242-9.

57. Hareyama M, Sakata K, Shirato H, et al. A prospective, randomized trial comparing neoadjuvant chemotherapy with radiotherapy alone in patients with advanced nasopharyngeal carcinoma. Cancer 2002;94:2217-23.

58. Agulnik M, Epstein JB. Nasopharyngeal carcinoma: Current management, future directions and dental implications. Oral Oncol 2008; $44: 617-27$.

59. Ang KK, Berkey BA, Tu X, et al. Impact of epidermal growth factor receptor expression on survival and pattern of relapse in patients with advanced head and neck carcinoma. Cancer Res 2002;62: 7350-6.

60. Chua DT, Nicholls JM, Sham JS, Au GK. Prognostic value of epidermal growth factor receptor expression in patients with advanced stage nasopharyngeal carcinoma treated with induction chemotherapy and radiotherapy. Int J Radiat Oncol Biol Phys 2004; 59:11-20.

61. Leong JL, Loh KS, Putti TC, et al. Epidermal growth factor receptor in undifferentiated carcinoma of the nasopharynx. Laryngoscope 2004;114:153-7.

62. Vered M, Braunstein E, Buchner A. Immunohistochemical study of epidermal growth factor receptor in adenoid cystic carcinoma of salivary gland origin. Head Neck 2002;24:632-6.

63. Ettl T, Schwarz S, Kleinsasser N, et al. Overexpression of EGFR and absence of C-KIT expression correlate with poor prognosis in salivary gland carcinomas. Histopathology 2008;53:567-77.

64. Laurie SA, Licitra L. Systemic therapy in the palliative management of advanced salivary gland cancers. J Clin Oncol 2006;24: 2673-8.

65. Ma B, Poon T, To KF, et al. Expression and prognostic significance of epidermal growth factor receptor and HER2 protein in nasopharyngeal carcinoma. Head Neck 2003;25:864-72.

66. Casalini P, Lorio MV, Galmozzi E, Menard S. Role of HER receptors family in development and differentiation. J Cell Physiol 2004;200: $343-50$.
67. Chua DT, Wei WI, Wong MP, et al. Phase II study of gefitinib for the treatment of recurrent and metastatic nasopharyngeal carcinoma. Head Neck 2008;30:863-7.

68. Ma B, Hui EP, King A, et al. A phase II study of patients with metastatic or locoregionally recurrent nasopharyngeal carcinoma and evaluation of plasma Epstein-Barr virus DNA as a biomarker of efficacy. Cancer Chemother Pharmacol 2008;62:59-64.

69. Chan AT, Hsu MM, Goh BC, et al. Multicenter, phase II study of cetuximab in combination with carboplatin in patients with recurrent or metastatic nasopharyngeal carcinoma. J Clin Oncol 2005; 23:3568-76.

70. You B, Le Tourneau C, Chen E, et al. A phase II trial of erlotinib after gemcitabine plus platinum-based chemotherapy in patients (pts) with recurrent and/or metastatic nasopharyngeal carcinoma (NPC). Eur J Cancer 2009;S7:478.

71. Ma BB, Leung SF, Kam MK, et al. A phase II study of concurrent cetuximab cisplatin and intensity-modulated radiotherapy (IMRT) in locoregionally advanced nasopharyngeal carcinoma (NPC) with correlation using dynamic contrast-enhanced magnetic resonance imaging (DCE-MRI). J Clin Oncol 2008;26:6055.

72. Krishna SM, James S, Balaram P. Expression of VEGF as prognosticator in primary nasopharyngeal cancer and its relation to EBV status. Virus Res 2006;115:85-90.

73. Segawa Y, Oda Y, Yamamoto H, et al. Close correlation between CXCR4 and VEGF expression and their prognostic implications in nasopharyngeal carcinoma. Oncol Rep 2009;21:1197-202.

74. Montag M, Dyckhoff G, Lohr J, et al. Angiogenic growth factors in tissue homogenates of HNSCC: expression pattern, prognostic relevance, and interrelationships. Cancer Sci 2009;100:1210-8.

75. Lothaire P, de Azambuja E, Dequanter D, et al. Molecular markers of head and neck squamous cell carcinoma: promising signs in need of prospective evaluation. Head Neck 2006;28:256-69.

76. Qian CN, Zhang CQ, Guo X, et al. Elevation of serum vascular endothelial growth factor in male patients with metastatic nasopharyngeal carcinoma. Cancer 2000;88:255-61.

77. Zhang G, Zeng J, Gong L, et al. Expression of vascular endothelial growth factor and nm23 as prognostic factors in nasopharyngeal carcinoma. Zhonghua Er Bi Yan Hou Ke Za Zhi 2001;36:372-5.

78. Zhao SP, Wang CL. Expression and clinical significance of vascular endothelial growth factor in nasopharyngeal carcinoma. Hunan Yi Ke Da Xue Xue Bao 2003;28:114-16.

79. Wakisaka N, Wen Q, Yoshizaki T, et al. Association of vascular endothelial growth factor expression with angiogenesis and lymph node metastasis in nasopharyngeal carcinoma. Laryngoscope 1999;109:810-4.

80. Kyzas PA, Cunha IW, Ionnidis JPA. Prognostic significance of vascular endothelial growth factor immunohistochemical expression in head and neck squamous cell carcinoma: A meta-analysis. Clin Cancer Res 2005;11:1434-40.

81. Druzgal CH, Chen Z, Yeah NT. A pilot study of longitudinal serum cytokine and angiogenesis factor levels as markers of therapeutic response and survival in patients with head and neck squamous cell carcinoma. Head Neck 2005;27:771-84. 